Revista de Psicología Vol. 31 (2), 2013 (ISSN 0254-9247)

\title{
Condicionantes ideológicos de la criminalización de la protesta social y el apoyo a la democracia en una muestra limeńa
}

\author{
Jan Marc Rottenbacher de Rojas ${ }^{1}$ y Mathias Schmitz ${ }^{2}$ \\ Pontificia Universidad Católica del Perú (PUCP)
}

Se analiza la influencia del conservadurismo político de derecha sobre el apoyo a la democracia y la criminalización de la protesta social en una muestra de estudiantes universitarios de Lima $(N=201)$. El conservadurismo político de derecha fue evaluado utilizando medidas de autoritarismo (RWA), orientación hacia la dominancia social (SDO) y orientación política de derecha. Dos diagramas de sendero (Path Analysis) fueron propuestos: el segundo diagrama presentó un ajuste adecuado y propone, en primer lugar, una covarianza positiva entre el RWA, la SDO y la orientación política de derecha. Asimismo, tanto el RWA, como la SDO y la orientación política de derecha ejercen una influencia directa sobre la criminalización de la protesta social, mientras solo la SDO y el RWA ejercen una influencia inversa sobre el apoyo a la democracia. Finalmente, se observa una correlación inversa entre el apoyo a la democracia y la criminalización de la protesta social.

Palabras clave: criminalización de la protesta, conservadurismo político, apoyo a la democracia.

\section{Ideological conditionings of the social protest criminalization and the support to democracy in a Lima sample}

The influence of right-wing political conservatism on support for democracy and the criminalization of social protest is analyzed in a sample of university students from the city of Lima $(N=201)$. As indicators of the right-wing political conservatism, measures of right-wing authoritarianism (RWA), social dominance orientation (SDO) and right-wing political orientation were used. Two path diagrams were proposed: the second diagram

1 Licenciado en Psicología Social y magíster en Historia por la PUCP. Profesor auxiliar del Departamento de Psicología de la PUCP y miembro del Grupo de Psicología Política de la misma Universidad. Ha publicado una serie de investigaciones empíricas en revistas especializadas de Brasil, Colombia, España, México y Perú. Dirección postal: Av. Universitaria 1801, San Miguel, Lima - 32, Perú. Dirección electrónica: jrottenbacher@pucp.edu.pe.

2 Licenciado en Psicología Social por la PUCP y miembro del Grupo de Psicología Política de la misma Universidad. Ha publicado investigaciones empíricas en revistas especializadas de Brasil, España y México. Dirección electrónica: mathias.schmitz@pucp.pe. 
showed a proper degree of fit and proposes a positive covariance between RWA, SDO and right-wing political orientation. The diagram also suggests that RWA, SDO and right-wing political orientation exert a direct influence on the criminalization of social protest, while SDO and RWA exert an indirect influence on support for democracy. Finally, support for democracy present an inverse correlation with the criminalization of social protest.

Key words: criminalization of protest, political conservatism, support for democracy 
El propósito general de esta investigación fue describir y analizar la influencia que podría ejercer un tipo de ideología denominado conservadurismo politico de derecha sobre dos actitudes sociopolíticas interrelacionadas: un fenómeno que ha sido denominado "criminalización de la protesta social" (Gamarra, 2010, p. 183) y el apoyo al sistema democrático. Sobre la base de la revisión bibliográfica que se presenta a continuación, se esperaba que los individuos con una tendencia hacia conservadurismo político de derecha mostrasen una actitud hostil hacia la protesta social, hasta el grado de percibirla como un tipo de crimen o delito que debe ser severamente castigado. Esta actitud hostil y criminalizante, se explica sobre el siguiente razonamiento: las protestas sociales representarían una amenaza contra la estabilidad y el orden social que los individuos políticamente más conservadores buscarían preservar.

\section{Las protestas sociales en el Perú actual (2001-2013)}

Luego de casi veinte años de crisis económica y tras haber atravesado el período más severo de violencia política de su historia —aproximadamente entre 1980 y 1998—, luego de la caída del régimen autoritario de Alberto Fujimori (1992-2000), el Perú experimentó en la última década (2001-2013) un sostenido crecimiento económico, acompañado de un proceso difícil, lento pero gradual, de consolidación del sistema democrático y del estado de derecho. Por un lado, el declive de la violencia terrorista desde mediados de la década de 1990, sumado a la progresiva consolidación de la democracia, ha otorgado a la población mayores espacios para manifestar sus demandas o insatisfacciones de manera pública. Según la Comisión de la Verdad y Reconciliación (2008), los años de violencia política promovieron la despolitización de la sociedad peruana, al contribuir a que todo aquel que protestara públicamente o se afiliara colectivamente con fines políticos, pudiera 
ser considerado subversivo y con ello, ser procesado como presunto terrorista y, en el peor de los casos, condenado a prisión por terrorismo. Por otro lado, el auge económico y la multiplicación de los proyectos de inversión en materia de minería y extracción de hidrocarburos generaron, entre los sectores menos favorecidos —en especial en las zonas rurales o periurbanas-, dos fenómenos antagónicos. Por un lado, grandes expectativas de inclusión y progreso económico y, por el otro, mucho temor con respecto al impacto negativo que las industrias extractivas podrían ejercer sobre su forma de vida, sus tradiciones, sus recursos naturales y sobre el medio ambiente en el que habitan (Defensoría del Pueblo, 2013).

Además de ello, la ineficiencia del sistema estatal para manejar adecuadamente estas expectativas y preocupaciones desencadenó la multiplicación de los conflictos sociales y las protestas colectivas en años recientes (Defensoría del Pueblo, 2012; Gamarra, 2010; Meléndez, 2004; Tanaka, Zárate \& Huber, 2011). De esta manera, de un promedio de 80 conflictos y 39 protestas sociales registradas el año 2006, se han reportado más de 220 conflictos y 95 acciones colectivas de protesta en lo que va del año 2013 (Defensoría del Pueblo, 2013, 2006). La gran mayoría de estos conflictos y protestas son de tipo socio-ambiental, cuya dinámica gira entrono al control, uso o acceso al medio ambiente natural y sus recursos, así como el impacto ambiental asociado a las actividades industriales extractivas (Defensoría del Pueblo, 2013). En un intento por desarticular estas protestas y mantener la expansión económica, el Estado y los sectores más privilegiados del país han recurrido a la criminalización de la protesta, entendida como "[...] la tipificación en las leyes penales, que realizan los Estados, de diversas modalidades de protesta, con el objetivo de combatirla, debilitarla o desorganizarla." (Salcedo, 2009, p. 93). Sin embargo, este incremento en las protestas no se ha limitado solo a los espacios rurales, sino que también ha aumentado el número de protestas en las ciudades, como producto de una serie de expectativas insatisfechas de diversos sectores de la población —en especial de los trabajadores del Estado o los sectores menos privilegiados- con respecto a las promesas de bienestar económico e inclusión 
social que son planteadas, tanto desde el Gobierno Central, como desde la propia lógica del crecimiento económico que prometen y difunden aquellos sectores que apoyan el sistema económico de libre-mercado que opera en el país desde la década de 1990.

\section{Criminalización de la protesta social}

Desde el punto de vista legal, la protesta social pacífica constituye un derecho ciudadano básico que se apoya sobre principios más generales como el derecho a la libertad de expresión, la libertad de reunión y el derecho a participar en la vida política del país (Gamarra, 2010). En este sentido, la criminalización de la protesta social implica el deseo de clasificar a este tipo de manifestaciones como "delitos atentatorios contra el orden público” (Gamarra, 2010, p. 183), lo cual justificaría no solo su represión, sino también la investigación penal de sus promotores o participantes. Algunos de los mecanismos mediante los cuales se manifiesta la criminalización de la protesta social serían: a) recurrir a la represión violenta, b) la expedición de normas que permiten el uso desproporcionado de la fuerza, d) la participación de las Fuerzas Armadas en el control de los conflictos sociales, e) recurrir al derecho penal para sancionarla, f) recurrir a la detención, investigación y procesamiento penal de manifestantes, y h) el hostigamiento hacia organizaciones o grupos que promuevan o lleven a cabo protestas sociales (Gamarra, 2010).

Siguiendo la propuesta de Linz (1978) es posible plantear que la criminalización de la protesta está estrechamente vinculada con aparatos políticos de tipo autoritario, ya que para este autor, el estilo autoritario de ejercicio del poder se caracteriza por el uso de la coerción, la limitación de la pluralidad política y la restricción de la participación política de la población. En la misma línea, y tomando como base las propuestas de Sartori (1988) y Linz (1978), se sugiere que para poder considerar a una determinada acción como autoritaria, esta debe cumplir por lo menos una de las siguientes tres condiciones: a) debe estar presente 
el uso de la fuerza o algún otro tipo de mecanismo coercitivo para llevarla a cabo, b) debe darse un mecanismo punitivo desproporcionado al momento de aplicar determinados castigos y c) debe aparecer, la limitación, cancelación o inexistencia de un espacio de negociación entre la población y la autoridad. De lo anterior, se deduce, en primer lugar, que el autoritarismo está estrechamente vinculado con la criminalización de la protesta social —y de la participación política en general—, y que criminalizar algún tipo de reclamo político implicaría: a) la legitimación del uso de la fuerza o algún otro tipo de mecanismo coercitivo para llegar a controlarlo, aplicar mecanismos punitivos desproporcionados para los involucrados en las protestas y, como producto de lo anterior, se produce la limitación o cancelación de un espacio de negociación entre la población y la autoridad.

\section{Conservadurismo político de derecha y criminalización de la protesta social}

Diversos estudios han encontrado, incluso en contextos latinoamericanos y de manera consistente, relaciones directas entre tres variables ideológicas: el autoritarismo de ala derecha — RWA, por sus siglas en inglés-, la orientación política de derecha y la orientación hacia la dominancia social —SDO, por sus siglas en inglés — (e. g., Altemeyer, 2004; Duckitt \& Sibley, 2007; Rottenbacher, 2012; Rottenbacher \& Schmitz, 2012a; Rottenbacher \& Schmitz, 2012b; Rottenbacher, Espinosa \& Magallanes, 2011; Rottenbacher, 2010; Sidanius, Pratto, Van Laar \& Levin, 2004). Dichas investigaciones sugieren que estas tres variables pueden considerarse como constituyentes de una tendencia ideológica más general, denominada por Thorisdottir, Jost, Liviatan y Shrout (2007, p. 178) como: "conservadurismo [político] de derecha”.

Las relaciones directas observadas entre el RWA, la SDO y la orientación política de derecha son consistentes con la propuesta conceptual de Bobbio (1996) en la cual una orientación hacia la derecha política expresa una justificación de la inequidad y un apoyo hacia las tradiciones o convencionalismo. En los estudios mencionados, la 
relación directa entre la SDO y la orientación política de derecha cobra sentido en la medida que la SDO expresa una tendencia general hacia la preferencia por sistemas sociales jerárquicos y la justificación o naturalización de la inequidad entre los grupos humanos (Altemeyer, 1998; Pratto, Sidanius, Stallworth \& Malle, 1994; Sidanius, Pratto, Van Laar \& Levin, 2004; Sidanius \& Pratto, 1999; Sidanius, Pratto \& Bobo, 1996). Por otro lado, la relación directa entre el RWA y una orientación política de derecha resulta coherente si se toma en cuenta que uno de los componentes actitudinales del RWA es el convencionalismo o defensa de las tradiciones (Altemeyer, 1981, 1988, 1998, 2004; Cottam, DietzUhler, Mastors \& Preston, 2004; Jost, Glaser, Kruglanski \& Sulloway, 2003). El RWA expresa tres tendencias actitudinales interrelacionadas: a) la sumisión autoritaria, que se manifiesta a través del apoyo hacia las instituciones y autoridades percibidas como legítimas y hacia las acciones o normas que estas promueven (Oesterreich, 2005), b) la agresión autoritaria, expresada a través del apoyo hacia métodos punitivos desproporcionados - e incluso violentos - contra los grupos que atentan contra dichas instituciones y autoridades, y, en general, contra la estabilidad y el orden social. Finalmente, el RWA también expresa convencionalismo, lo que se traduce en la defensa y la intención de preservar las tradiciones o convenciones sociales más tradicionales (Altemeyer, 1981, 1988, 1998, 2004; Cottam, Dietz-Uhler, Mastors \& Preston, 2004; Jost et al., 2003).

Con respecto a la SDO, ella representa una visión jerárquica de la sociedad en la que algunos grupos - generalmente el endogrupoposeen mayor valor o prestigio que otros. Una mayor tendencia hacia la SDO se expresa a través de la naturalización de las diferencias jerárquicas al interior de una sociedad y la justificación de la inequidad económica y social (Altemeyer, 1998; Pratto et al., 1994; Sidanius et al., 2004; Sidanius \& Pratto, 1999; Sidanius, Pratto \& Bobo, 1996). La utilización conjunta de estas dos variables — RWA y SDO — ha sido denominada como el enfoque dual de la ideología política (Duckitt \& Sibley, 2007; Duckitt \& Fisher, 2003; Duckitt, Wagner, du Plessis \& Birum, 2002; Jost, Federico \& Napier, 2009; Van Hiel \& Mervielde, 2002). 
Para Jost y colaboradores (2003), el conservadurismo político debe entenderse como una forma de cognición social motivada, en la cual, las denominadas necesidades epistémicas y las necesidades existenciales condicionan la adhesión de las personas hacia posturas ideológicas conservadoras. Las necesidades epistémicas están relacionadas con el nivel de orden, estructuración, incertidumbre y ambigüedad que las personas son capaces de tolerar. Por otro lado, las necesidades existenciales están asociadas a los niveles de inseguridad real o simbólica que las personas son capaces de soportar. Desde esta perspectiva teórica, todo estímulo o contexto social que eleve los niveles de incertidumbre y amenaza percibida, favorecerá en general, la inclinación de las personas hacia posturas y acciones políticamente conservadoras (Jost et al., 2003) y hasta autoritarias (Oesterreich, 2005), con la finalidad de reducir la incertidumbre e incrementar la sensación de seguridad. Desde la perspectiva de Jost y colaboradores (2003), es posible proponer que las protestas sociales - manifestaciones públicas, marchas, mítines, huelgas, entre otras - pueden elevar los niveles de incertidumbre y amenaza que las personas con una mayor tendencia hacia el conservadurismo político pudieran ser capaces de tolerar. En este sentido, la criminalización de la protesta social, serviría como estrategia para reducir estos niveles de incertidumbre y amenaza, al clasificar a todo aquel que participe en ellas como delincuente y merecedor, por ello, de castigos y penas similares a las de los criminales. Finalmente, es posible sostener que la criminalización de la protesta social satisface las necesidades epistémicas propuestas por Jost y colaboradores (2003), al ofrecer certezas - por lo menos asumidas como verdades por los individuos más conservadores-acerca de los orígenes, motivos y formas de resolución de dichas protestas públicas.

A partir de la revisión bibliográfica presentada, esta investigación se propuso indagar acerca de la influencia que el conservadurismo político de derecha — evaluado a través del RWA, la SDO y la orientación política de derecha- podría ejercer sobre dos actitudes sociopolíticas: el apoyo a la democracia y la criminalización de la protesta social. Se plantean las siguientes hipótesis: debería observarse una correlación 
directa entre los componentes del conservadurismo político —el RWA, la SDO y la orientación política de derecha- $\left(H_{1}\right)$. Estos componentes correlacionados directamente entre sí, deberían ejercer un efecto negativo sobre el apoyo a la democracia $\left(H_{2}\right)$ y, a la vez, un efecto positivo sobre la criminalización de la protesta social $\left(H_{3}\right)$. A partir de las tres primeras hipótesis se deriva una cuarta hipótesis $\left(H_{4}\right)$, en la que debería observase una correlación negativa entre el apoyo a la democracia y la criminalización de la protesta social

\section{Método}

\section{Participantes}

Se utilizó un muestreo de tipo no probabilístico intencional (Hernández, Fernández \& Baptista, 2007), recurriendo a las personas que frecuentan el campus de una universidad privada de Lima. La muestra final $(N=201)$ estuvo conformada por adultos cuyas edad fluctuaron entre un mínimo de 18 y un máximo de 40 años $(M E=24.6, D E=4.4)$, siendo la mayoría hombres $(54.9 \%)$. Con respecto a su nivel educativo, la mayoría eran estudiantes universitarios (55.2\%), o bien egresados de alguna universidad (22.9\%). Un 7.5\% se encontraba estudiando alguna carrera técnica, un $7.0 \%$ ya la había culminado, un $4.0 \%$ contaba con estudios de posgrado y solo un $3.5 \%$ contaba únicamente con estudios escolares completos. La mayoría (57.8\%) se ubicó en un nivel socioeconómico (NSE) medio, el $26.9 \%$ en un NSE medio-alto, el $9.0 \%$ en un NSE medio-bajo, el $4.5 \%$ en un NSE alto, y solo el $1.0 \%$ en un NSE bajo. En general, los participantes provenían de 29 distritos de la ciudad de Lima Metropolitana y el puerto de El Callao.

\section{Instrumentos}

Autoritarismo de Ala Derecha (RWA). Se utilizó una versión traducida al castellano por Rottenbacher y Schmitz (2012a) de la versión de la Escala Resumida de Autoritarismo de Ala Derecha de Zakrisson (2005). 
Este instrumento está compuesto por 15 ítems, como por ejemplo: "Nuestro país necesita un líder poderoso para erradicar las tendencias radicales e inmorales prevalentes en la sociedad actual", o "El guiarse por los valores tradicionales sigue siendo la manera más adecuada de vivir". La opción de respuesta se presentó en una escala Likert de 6 puntos donde: 1 = "Totalmente en Desacuerdo" y 6 = "Totalmente de Acuerdo". El instrumento presentó un adecuado nivel de confiabilidad $(\alpha=.79)$ de acuerdo con los criterios establecidos por Mezulis, Abramson, Hyde y Hankin (2004).

Orientación Política de Derecha. Se utilizó un solo ítem cuya consigna fue: "Normalmente, en términos de orientación o simpatías políticas la gente se ubica en un continuo que va desde la Izquierda hasta la Derecha. Quisiéramos que haga el ejercicio para ubicarse usted mismo el ese continuo según la siguiente escala donde: 1 = 'De izquierda' y 6 = 'De derecha.'". Con respecto a este ítem, un puntaje mayor a la media teórica $(M T=3.5)$ expresa una auto-clasificación política orientada hacia la derecha ideológica.

Orientación hacia la Dominancia Social (SDO). Se utilizó la versión traducida al castellano por Montes-Berges y Silván-Ferrero (citados en Moya \& Morales-Marente, 2005) de la Escala de Orientación hacia la Dominancia Social (SDO de Sidanius \& Pratto citado en Sidanius et al., 2004). Esta escala comprende 16 enunciados referidos a relaciones jerárquicas y asimétricas entre grupos pertenecientes a una misma sociedad. La opción de respuesta es una escala Likert de 6 puntos donde: 1 = "Totalmente en Desacuerdo" y 6 = "Totalmente de Acuerdo". Este instrumento presentó un alto nivel de confiabilidad para el caso del presente estudio $(\alpha=.84)$.

Apoyo a la Democracia. Se utilizó el cuestionario sobre Apoyo a la Democracia utilizado por la Corporación Latinobarómetro (2009). El cuestionario está compuesto de 13 ítems cuya opción de respuesta es una escala Likert de 6 puntos donde: 1 = "Totalmente en Desacuerdo" y 6 = "Totalmente de Acuerdo". La escala de 13 ítems presentó un adecuado nivel de confiabilidad $(\alpha=.77)$. 
Criminalización de la Protesta Social. Debido a la ausencia de instrumentos para evaluar esta variable, se consideró pertinente elaborar un instrumento ad hoc de 20 ítems (ver Anexo A) cuya opción de respuesta es una escala Likert de 6 puntos donde: 1 = "Totalmente en Desacuerdo" y 6 = "Totalmente de Acuerdo". Los ítems eran similares a: "Se debe castigar duramente a quienes protestan públicamente y alteran con ello la paz social", o "Todo ciudadano tiene derecho a protestar públicamente si no está de acuerdo con algo”. Los ítems de la Escala de Criminalización de la Protesta Social fueron sometidos a una evaluación por tres jueces expertos, todos psicólogos sociales, quienes luego de revisarlos, no rechazaron ninguno de los ítems, sino que sugirieron pequeñas modificaciones de redacción. La escala de 20 ítems obtuvo un nivel de confiabilidad alto $(\alpha=.90)$.

\section{Procedimiento}

Se contactó a los participantes en el campus de una universidad privada de Lima. A todos se les pidió su colaboración voluntaria y en todo momento se les aseguró el anonimato de sus respuestas y la completa libertad de dejar de llenar el cuestionario si así lo deseaban. A las personas que accedieron a participar se les leyó el consentimiento informado y se les entregó el cuestionario de autorreporte que contenía todos los instrumentos para la evaluación de las variables del estudio. Los datos fueron recogidos entre octubre y noviembre de 2012. La información fue analizada por los investigadores utilizando los programas estadísticos $\operatorname{SPSS}^{\odot} 21$ y $\operatorname{AMOS}^{\odot} 21$.

\section{Resultados}

Con respecto al puntaje promedio de las variables, se observa que el Apoyo a la Democracia presentó la media más alta $(M=4.51)$, seguida por la Orientación Política de Derecha $(M=3.62)$. Por debajo de la media teórica $(M T=3.50)$ se encuentran, en orden descendente, 
el RWA, la Criminalización de la Protesta Social $^{3}$ y, finalmente, la SDO (ver Tabla 1). Asimismo, se puede apreciar que todas las correlaciones resultaron significativas, a excepción de la correlación entre el Apoyo a la Democracia y la Orientación Política de Derecha.

Por otro lado, los tres componentes del conservadurismo político de derecha —el RWA, la SDO, y la Orientación Política de Derecha- se relacionan entre sí de manera directa. El RWA y la SDO correlacionan ambos, de manera directa, con la Criminalización de la Protesta Social y de forma negativa con Apoyo a la Democracia. Finalmente, la Criminalización de la Protesta Social correlaciona de manera inversa con el Apoyo a la Democracia.

Tabla 1. Matriz de Correlaciones entre las variables de estudio

\begin{tabular}{|c|c|c|c|c|c|c|c|}
\hline & 1 & 2 & 3 & 4 & 5 & $M$ & $D E$ \\
\hline $\begin{array}{l}\text { 1. Criminalización de la } \\
\text { Protesta Social }\end{array}$ & - & $.35^{* *}$ & $.52^{* *}$ & $.38^{* *}$ & $-.35^{* *}$ & 2.50 & 0.84 \\
\hline 2. SDO & & - & $.31^{* *}$ & $.25^{* *}$ & $-.45^{* *}$ & 2.24 & 0.75 \\
\hline 3. RWA & & & - & $.34^{* *}$ & $-.31^{* *}$ & 2.95 & 0.78 \\
\hline $\begin{array}{l}\text { 4. Orientación Política de } \\
\text { Derecha }\end{array}$ & & & & - & -.09 & 3.62 & 1.20 \\
\hline 5. Apoyo a la Democracia & & & & & - & 4.51 & 0.74 \\
\hline${ }^{*} p<.05 ;{ }^{* *} p<.01$ & & & & & & & \\
\hline
\end{tabular}

A partir de las hipótesis del estudio se elaboró el diagrama hipotético, denominado Modelo 1 (ver Figura 1). Este diagrama no obtuvo indicadores de ajuste suficientemente buenos, según los criterios propuestos por diversos autores (e. g., Bollen, 1989; Kline, 2005; Ruiz, Pardo \& San Martín, 2010; Schumacker \& Lomax, 2004): $\chi^{2}(1)=6.801, p=.009, \chi^{2} / g l=6.801, \mathrm{CFI}=.96, \mathrm{NFI}=.96, \mathrm{TLI}=.68$, $\mathrm{IFI}=.97, \mathrm{RFI}=.64, \mathrm{RMSEA}=.17(90 \% \mathrm{CI}=.07 \leq .17 \leq .30)$. Además,

3 Para observar el comportamiento muestra de esta variable se presenta en el Anexo B un histograma que grafica las frecuencias observadas con respecto a la Criminalización de la Protesta Social. 
la relación de influencia inversa que la Orientación Política de Derecha debía ejercer sobre el Apoyo a la Democracia no resultó significativa.

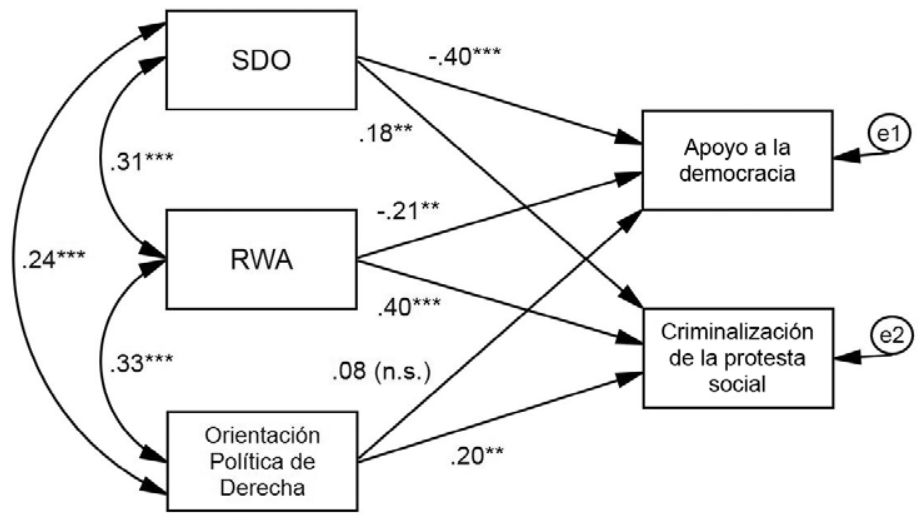

Figura 1. Diagrama Hipotético (Modelo 1)

${ }^{* * *} p<.001 ;{ }^{* *} p<.01$

Por estos motivos, se eliminó la relación no significativa y se propuso un diagrama alternativo, denominado Modelo 2 (ver Figura 2). Este diagrama obtuvo buenos indicadores de ajuste. En primer lugar, según los valores del estadístico Chi-cuadrado, se puede sostener que todos los errores del diagrama son iguales a cero: $\chi^{2}(1)=1.506, p=$ .220. Asimismo, los demás indicadores de ajuste también resultaron suficientemente adecuados $\chi^{2} / g l=1.506, \mathrm{CFI}=.99, \mathrm{NFI}=.99, \mathrm{TLI}=$ $.97, \mathrm{IFI}=.99$, RFI $=.92$, RMSEA $=.05(90 \% \mathrm{CI}=.001 \leq .05 \leq .20)$, $\mathrm{y}$ todas las relaciones planteadas en el diagrama resultaron significativas (ver Figura 2).

4 Debido a que, tanto el Apoyo a la Democracia como la Criminalización de la Protesta Social reciben la influencia de las mismas variables independientes, se asume una covarianza entre estas dos variables. Sin embargo, esta correlación no puede colocarse en el diagrama porque conduce a que sea imposible calcular sus estadísticos de ajuste. 


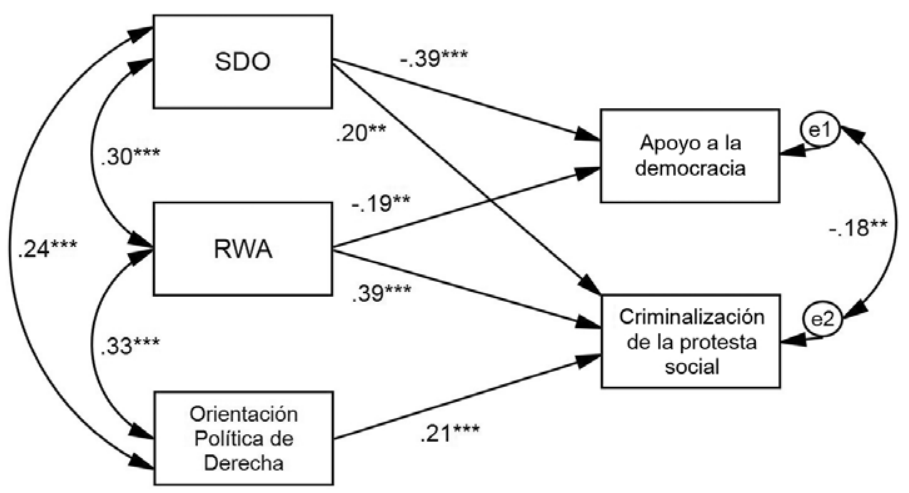

Figura 2. Diagrama Alternativo (Modelo 2)

${ }^{* * *} p<.001 ;{ }^{* *} p<.01$

\section{Discusión}

En términos generales, el supuesto inicial de este estudio ha podido ser corroborado a través de los resultados. Por un lado, una mayor inclinación hacia el conservadurismo político de derecha ejerce un efecto inverso sobre el apoyo al sistema democrático y, por el otro, un efecto directo sobre la criminalización de la protesta social. Si bien las hipótesis específicas planteadas en el Modelo 1 no llegaron a corroborarse del todo debido a que los indicadores de ajuste del diagrama no resultaron adecuados, en el Modelo 2 que modifica ligeramente las hipótesis iniciales, se ha logrado arribar a resultados similares a los que se había previsto. A continuación se discute con mayor grado de detalle, las relaciones de influencia que se han encontrado entre las variables de esta investigación.

En primer lugar, a nivel descriptivo, los resultados muestran que los participantes de este estudio se caracterizan por presentar un apoyo bastante elevado hacia el sistema democrático $(M=4.51)$ y se autodefinen como de una orientación política de derecha $(M=3.62)$. Esto debido a que las medias en estas dos variables superan la media teórica 
que es, según la escala de respuesta de los instrumentos utilizados de 3.5 puntos. Por otro lado, la muestra presenta niveles moderados en RWA y niveles relativamente bajos en la SDO. Estos resultados son coherentes con estudios previos realizados en muestras con características similares en lo que los niveles de RWA siempre son mayores a los observados en la SDO (e. g., Espinosa, 2011; Rottenbacher, 2012; Rottenbacher \& De la Cruz, 2012; Rottenbacher \& Schmitz 2012a, $2012 b)$. Esto último sugiere que los estudiantes universitarios evaluados en el presente estudio podrían ser catalogados, desde un punto de vista ideológico-político, como personas prodemocráticas de derecha, que si bien tienden hacia el autoritarismo, no pueden ser clasificadas como autoritarias y tampoco tienden a justificar la inequidad social, porque sus niveles en la SDO no son muy elevados. Con respecto a la criminalización de la protesta social, el puntaje promedio de esta variable es bajo $(M=2.50)$, pero no tanto como para sostener que a nivel muestral existe un rotundo rechazo hacia la criminalización de la protesta social. En el Anexo B se observa que, si bien existe una tendencia hacia el rechazo de la criminalización de la protesta social, hay un importante grupo de la muestra que presenta puntajes mayores a 3.0 e, incluso, a 3.5 , lo que expresa una variabilidad importante con respecto a la actitud de los participantes frente a este fenómeno. Las protestas sociales son aceptadas por la mayoría de los participantes, quienes rechazan su criminalización, pero hay un porcentaje importante (28.4\%) que presenta puntuaciones por encima de los 3 puntos, quienes no estarían tan en desacuerdo con que la criminalización de la protesta social pudiera ser una medida gubernamental positiva.

A continuación se discuten los resultados a la luz de las cuatro hipótesis del estudio que se han enfocado en las relaciones entre las variables analizadas. En primer lugar, las covarianzas -o correlaciones- positivas que se observan en el Modelo 2 entre el autoritarismo de ala derecha (RWA), la orientación hacia la dominancia social (SDO) y la orientación política de derecha, corroboran la primera hipótesis del estudio $\left(H_{1}\right)$, que proponía que el RWA, la SDO y la orientación política de derecha debían estar relacionadas entre sí de manera 
directa. Estos resultados nos permiten sostener que estas tres variables, utilizadas de manera conjunta, son buenos indicadores de un tipo de ideología política, que como ha sido mencionado, Thorisdottir et al. (2007) denominaron conservadurismo político de derecha.

En segundo lugar, se esperaba que estos tres componentes del conservadurismo político ejercieran una influencia inversa sobre el apoyo al sistema democrático. Esto, en la medida en que el conservadurismo político expresa, entre otras actitudes, una tendencia hacia la justificación de la inequidad y el autoritarismo (Bobbio, 1996; Jost et al., 2003; Jost et al., 2009). Estas dos tendencias — autoritarismo y justificación de la inequidad - serían incompatibles con el funcionamiento pleno de una democracia en la medida en que esta se asocia a valores como la inclusión y equidad social, el trato igualitario entre las personas, la participación de las mayorías y las minorías, la libertad de opinión, el respeto por las diferencias culturales, etc. En este sentido, los resultados de este estudio corroboran parcialmente esta segunda hipótesis $\left(H_{2}\right)$ en la medida en que, si bien el RWA y la SDO ejercen un efecto negativo sobre el apoyo a la democracia, no se observa lo mismo en el caso de la orientación política de derecha. Este último resultado adquiere sentido si se toma en cuenta que una orientación moderada hacia la derecha política no necesariamente implicaría un rechazo hacia la democracia o a sus reglas de juego. Por el contrario, es al interior de un sistema democrático donde las fuerzas políticas moderadas - ya sea que se consideren de derecha o de izquierda - son capaces de desarrollar sus propias actividades políticas. Para Bobbio (1996), el autoritarismo y, por lo tanto, el rechazo hacia formas más democráticas de ejercicio del poder, solo se presenta en los extremos ideológicos de derecha o izquierda. Por ello, es coherente que este estudio haya encontrado que tanto el RWA que expresa autoritarismo y la SDO que expresa una tendencia hacia la justificación de la inequidad ejerzan, ambos, una influencia inversa sobre el apoyo a la democracia. Esta influencia es de mayor intensidad en el caso de la SDO y menor en el caso del RWA (ver Figura 2). 
En tercer lugar, se esperaba que los tres componentes del conservadurismo político de derecha - RWA, SDO y orientación política de derecha- ejercieran una influencia directa sobre la criminalización de las protestas sociales $\left(H_{3}\right)$. Los resultados obtenidos corroboraron esta hipótesis. Estos hallazgos están en correspondencia con el razonamiento de que los individuos políticamente más conservadores son menos tolerantes a la incertidumbre, la ambigüedad y los cambios sociales abruptos, por lo que prefieren un mundo social estructurado, estable y predecible (Jost et al., 2009; Jost et al., 2003; Rottenbacher, 2012). Por ello, es lógico pensar que estas personas mostrarían un cierto nivel de rechazo frente a las protestas sociales — cuyas manifestaciones son públicas pero no necesariamente violentas - dado que, de alguna u otra forma, buscan generar un cambio en la organización social o en un aspecto específico de esta. Con la finalidad de retornar al orden y estabilidad social, la criminalización o penalización de la protesta social aparece como una estrategia psicológica que devuelve a los individuos políticamente más conservadores, certidumbre, tranquilidad y claridad acerca del entorno social. Así, las personas involucradas en las protestas son concebidas como criminales o delincuentes, merecedores de penas muy altas que incluyen la reclusión carcelaria en su versión más extrema.

Por otra parte, se ha propuesto que los individuos más conservadores, cuando son expuesto a este tipo de situaciones, suelen exhibir la denominada repuesta autoritaria (Oesterreich, 2005) o agresión autoritaria (Altemeyer, 1981, 1988, 1998, 2004), es decir, un apoyo hacia las autoridades percibidas como legítimas, en tanto se encarguen de preservar el orden social, y una predisposición a aceptar mecanismos punitivos desproporcionados, si estos están encaminados hacia la restauración de la estabilidad social. Tomando en cuenta ello, es posible sostener que la criminalización de la protesta social constituiría un claro ejemplo de respuesta o agresión autoritaria en la medida que implica la aplicación de castigos penales y el tratamiento delincuencial de una práctica social que, en la mayor parte de las democracias occidentales, es un derecho básico de las personas. Por consiguiente, se puede deducir que la criminalización de la protesta social es una expresión de autoritarismo. 
Respecto a la última hipótesis de este estudio, se corrobora la relación negativa que se propuso, debía observarse entre el apoyo al sistema democrático y la criminalización de la protesta social $\left(H_{4}\right)$. Ello es congruente con la idea que la criminalización de la protesta social es percibida como un atentado a los derechos de expresión, reunión y participación política sobre los cuales se funda la democracia (Gamarra, 2010; Catholic International Cooperation for Development and Solidarity (CIDSE), 2011). Como ya fue mencionado, en términos generales, el supuesto teórico que guió esta investigación ha podido ser corroborado a través de los resultados obtenidos y se ha podido arribar, además, a un análisis más detallado de la relación entre el conservadurismo político de derecha y su relación con la criminalización de una manifestación propia de los sistemas políticos democráticos: la protesta social.

Como toda investigación que intenta recoger evidencia empírica de un fenómeno poco estudiado en la realidad peruana y latinoamericana, los resultados de esta investigación, así como sus limitaciones, deberían ser tomados como puntos de partida para el diseño de estudios más metodológicamente más ambiciosos que corroboren, amplíen o cuestionen los hallazgos del presente estudio. Como reflexión final y aplicando los resultados obtenidos al contexto social peruano, sobre la base de los informes de la Defensoría del Pueblo (2013) es posible proponer que las propuestas sociales puedan ir en aumento en los próximos años y que, de ser así, es probable que desde los diversos poderes del Estado Peruano, pudieran proponerse normas o medidas concretas de corte autoritario dirigidas hacia la criminalización de dichas protestas. En este escenario hipotético y según lo observado en este estudio, algunos sectores de la población - aquellos con una mayor tendencia hacia el conservadurismo político de derecha - podrían ser proclives hacia el apoyo de estas medidas, en un intento por preservar su sensación de orden y estabilidad en el entorno social. Finalmente, es posible proponer que a lo largo de la historia peruana, las medidas punitivas desproporcionadas no han resultado ser, en ningún escenario y bajo ninguna condición, estrategias adecuadas para la resolución de los conflictos, sino que, tarde o temprano han contribuido a intensificarlos. 


\section{Referencias}

Altemeyer, B. (1981). Right-wing authoritarianism. Winnipeg: University of Manitoba.

Altemeyer, B. (1988). Enemies of freedom: Understanding right-wing authoritarianism. San Francisco: Jossey-Bass.

Altemeyer, B. (1998). The other "authoritarian personality". En M. Zanna (Ed.), Advances in experimental social psychology, 30, 47-91. Nueva York: Academic Press.

Altemeyer, B. (2004). Highly dominating, highly authoritarian personalities. The Journal of Social Psychology, 144(4), 421-447.

Bobbio, N. (1996). Derecha e izquierda. Razones y significados de una distinción politica. Madrid: Taurus.

Bollen, K. (1989). A New Incremental Fit Index for General Structural Equation Models. Sociological Methods \& Research, 17(3), 303-316.

Catholic International Cooperation for Development and Solidarity (CIDSE) (2011). La criminalización de la protesta social en torno a la industria extractiva en América Latina. Recuperado el 11 de setiembre de 2013 de http:/www.cidse.org/content/ publications/business-a-human-rights/extractive-industries-inlatin-america/criminalisation_social_protest_latin_america.html Comisión de la Verdad y Reconciliación. (2008). Hatun Willakuy. Versión abreviada del Informe Final de la Comisión de la Verdad y Reconciliación. Lima: Comisión de Entrega de la CVR.

Corporación Latinobarómetro (2009). Informe 2009. Recuperado el 25 de febrero de 2013 de http://www.asep-sa.org/latinobarometro/ LATBD_Latinobarometro_Informe_2009.pdf

Cottam, M., Dietz-Uhler, B., Mastors, E. \& Preston, T. (2004). Introduction to Political Psychology. Nueva Jersey: Lawrence Erlbaum Associates.

Defensoría del Pueblo (2006). Reporte $N^{\circ}$ 23. Conflictos sociales conocidos por la Defensoría del Pueblo al 31 de enero de 2006. Lima: Defensoría del Pueblo.

Defensoría del Pueblo (2012). Resolución defensorial $N^{\circ}$ 009-2012/DP. Lima: Defensoría del Pueblo. 
Defensoría del Pueblo (2013). Reporte de conflictos sociales $N^{\circ} 112$. Lima: Defensoría del Pueblo.

Duckitt, J., Wagner, C., du Plessis, I. \& Birum, I. (2002). The psychological bases of ideology and prejudice: Testing a dual process model. Journal of Personality and Social Psychology, 83, 75-93.

Duckitt, J. \& Fisher, K. (2003). The impact of social threat on worldview and ideological attitudes. Political Psychology, 24, 199-222.

Duckitt, J. \& Sibley, C. (2007). Right Wing Authoritarianism, Social Dominance Orientation and the Dimensions of Generalized Prejudice. European Journal of Personality, 21, 113-130.

Espinosa, A. (2011). Estudios sobre identidad nacional en el Perú y sus correlatos psicológicos, sociales y culturales. Tesis doctoral. Universidad del País Vasco-Euskal Herriko Unibertsitatea, País Vasco.

Gamarra, R. (2010). Libertad de expresión y criminalización de la protesta social. En E. A. Bertonia, ¿Es legítima la criminalización de la protesta social?: derecho penal y libertad de expresión en América Latina (pp. 183-208). Buenos Aires: Universidad de Palermo.

Hernández, R., Fernández, C. \& Baptista, P. (2007). Metodología de la investigación ( $4^{\mathrm{a}} \mathrm{ed}$.). México: McGraw-Hill Interamericana.

Jost, J., Glaser, J., Kruglanski, A. \& Sulloway, F. (2003). Political Conservatism as Motivated Social Cognition. Psychological Bulletin, 129(3), 339-375.

Jost, J., Federico, C. \& Napier, J. (2009). Political ideology: Its structure, functions, and elective affinities. Annual Review of Psychology, 60, 307-337.

Kline, R. (2005). Principles and practice of structural equation modelling (2da. edición). Nueva York: Guilford.

Linz, J. J. (1978). Una Interpretación de los Regímenes Autoritarios. Revista de Sociología, 8, 11-26.

Meléndez, C. (2004). Hipótesis sobre los conflictos en el Perú actual. Lima: Asociación Civil Transparencia.

Mezulis, A., Abramson, L., Hyde, J. \& Hankin, B. (2004). Is there a universal positivity bias in attributions? A meta-analytic review of individual, developmental and cultural differences in the selfserving attributional bias. Psychological Bulletin, 130(5), 711-747. 
Moya, M. \& Morales-Marente, E. (2005). Reacciones psico-políticas ante los ataques terroristas del 11 de marzo de 2004. Revista de Psicología Social, 20, 331-350.

Oesterreich, D. (2005). Flight into security: A new approach and measure of the authoritarian personality. Political Psychology, 26, 275-297.

Pratto, J., Sidanius, J., Stallworth, L. \& Malle, B. (1994). Social dominance orientation: A personality variable predicting social and political attitudes. Journal of Personality and Social Psychology, 67, 741-763.

Rottenbacher, J. M. (2012). Conservadurismo político y rigidez cognitiva en una muestra de estudiantes y egresados universitarios de la ciudad de Lima. Avances en Psicología Latinoamericana, 30(2), 257-271.

Rottenbacher, J. M. y De la Cruz, M. F. (2012). Ideología política y actitudes hacia la minería en el Perú: entre el crecimiento económico, el respeto por las formas de vida tradicionales y el ambientalismo. Liberabit, 18(1), 83-96.

Rottenbacher, J. M. \& Schmitz, M. (2012a). Conservadurismo político y tolerancia hacia comportamientos transgresores. Revista Psicología Politica de la Universidad de Valencia, 44, 31-56.

Rottenbacher, J. M. \& Schmitz, M. (2012b). Democracia vs neoliberalismo económico. Condicionantes ideológicos de las preferencias políticas y económicas en la ciudad de Lima. Revista Mexicana de Ciencias Politicas y Sociales, 52(214), 111-140.

Rottenbacher, J. M., Espinosa, A. \& Magallanes, J. M. (2011). Analizando el prejuicio: bases ideológicas del racismo, el sexismo y la homofobia en una muestra de habitantes de la ciudad de Lima-Perú. Revista Psicologia Política de la Associação Brasileira de Psicologia Politica, 11(22), 225-246.

Rottenbacher, J. M. (2010). Sexismo ambivalente, paternalismo masculino e ideología política en adultos jóvenes de la ciudad de Lima. Revista Pensamiento Psicológico, 7(14), pp. 9-18.

Ruiz, M., Pardo, A. \& San Martín, R. (2010). Modelos de Ecuaciones Estructurales. Papeles del Psicólogo, 31(1), 34-45. 
Salcedo, C. M. (2009). El derecho constitucional de reunión y la protesta social. Gaceta Constitucional, 19, 83-96.

Sartori, G. (1988). Teoría de la democracia. Madrid: Alianza Editorial.

Schumacker, R. \& Lomax, R. (2004). A beginner's guide to structural equation modeling (2da. edición). Mahwah, NJ: Erlbaum

Sidanius, J., Pratto, F. \& Bobo, L. (1996). Racism, conservatism, affirmative action, and intellectual sophistication: A matter of principled conservatism or group dominance? Journal of Personality and Social Psychology, 70, 476-490.

Sidanius, J. \& Pratto, F. (1999). Social dominance: An intergroup theory of social hierarchy and oppression. Nueva York: Cambridge University Press.

Sidanius, J., Pratto, F., Van Laar, C. \& Levin, S. (2004). Social dominance theory: Its agenda and method. Political Psychology, 25(6), 845-880.

Tanaka, M., Zárate, P. \& Huber, L. (2011). Mapa de conflictividad social en el Perú: Análisis de sus principales causas. Lima: Biblioteca Nacional del Perú.

Thorisdottir, H., Jost, J., Liviatan, I. \& Shrout, P. (2007). Psychological Needs and Values Underlying Left-Right Political Orientation: Cross-National Evidence from Eastern and Western Europe. Public Opinion Quarterly, 71(2), 175-203.

Van Hiel, A. \& Mervielde, I. (2002). Explaining conservative beliefs and political preferences: A comparison of social dominance orientation and authoritarianism. Journal of Applied Social Psychology, 32, 965-976.

Zakrisson, I. (2005). Construction of a short version of the RightWing Authoritarianism (RWA) scale. Personality and Individual Differences, 39, 863-872.

Recibido: 23 de septiembre, 2013 Aceptado: 11 de octubre, 2013 


\section{Anexo A}

Tabla 2. Items y estadísticos de confiabilidad de la escala para evaluar la "Criminalización de la protesta social"

\begin{tabular}{|c|c|c|c|}
\hline Ítems & $\begin{array}{l}\text { Correlación } \\
\text { ítem-test } \\
\text { corregida }\end{array}$ & $\begin{array}{l}\text { Alfa de } \\
\text { Cronbach } \\
\text { si se elimina } \\
\text { el ítem }\end{array}$ & $\begin{array}{l}\text { Alfa de } \\
\text { Cronbach } \\
\text { de la escala } \\
\text { completa }\end{array}$ \\
\hline $\begin{array}{l}\text { 1. Todo ciudadano tiene derecho a protestar públicamente } \\
\text { si no está de acuerdo con algo. (Inverso) }\end{array}$ & .34 & .90 & \\
\hline $\begin{array}{l}\text { 2. Se debe castigar duramente a quienes protestan } \\
\text { públicamente y alteran con ello la paz social. }\end{array}$ & .60 & .90 & \\
\hline $\begin{array}{l}\text { 3. En el ejercicio de su deber, la Policía tiene todo el } \\
\text { derecho de usar la fuerza contra las personas que protestan } \\
\text { públicamente. }\end{array}$ & .67 & .90 & \\
\hline $\begin{array}{l}\text { 4. Se debe castigar a los miembros de la Policía que en el } \\
\text { ejercicio de su deber, hacen daño o causan la muerte de } \\
\text { participantes en protestas públicas. (Inverso) }\end{array}$ & .32 & .90 & \\
\hline $\begin{array}{l}\text { 5. La Policía debería actuar con "mano dura" para resolver } \\
\text { los conflictos sociales y restablecer el orden. }\end{array}$ & .52 & .90 & \\
\hline $\begin{array}{l}\text { 6. La Policía debería intervenir en favor de las empresas } \\
\text { privadas cuando se da una situación de conflicto social. }\end{array}$ & .51 & .90 & \\
\hline $\begin{array}{l}\text { 7. Se debería dar una ley para que la Policía brinde su } \\
\text { apoyo a las empresas privadas cuando existen situaciones } \\
\text { de protesta pública. }\end{array}$ & .61 & .90 & \\
\hline 8. Cuando ya no se puede negociar, es necesario que las & & & .90 \\
\hline $\begin{array}{l}\text { Fuerzas Armadas intervengan para reprimir las protestas } \\
\text { públicas. }\end{array}$ & .52 & .90 & \\
\hline $\begin{array}{l}\text { 9. Las Fuerzas Armadas deben asegurar el orden interno } \\
\text { usando todos los medios y recursos que sean necesarios. }\end{array}$ & .62 & .89 & \\
\hline $\begin{array}{l}\text { 10. Siempre se debería recurrir a las Fuerzas Armadas para } \\
\text { el control de los conflictos sociales. }\end{array}$ & .62 & .90 & \\
\hline $\begin{array}{l}\text { 11. La protesta pública debe ser considerada un delito } \\
\text { grave y se deben establecer penas de cárcel para las } \\
\text { personas que protesten públicamente. }\end{array}$ & .63 & .90 & \\
\hline $\begin{array}{l}\text { 12. El Estado debería castigar con la privación de la libertad } \\
\text { a todo aquel que al protestar, altere el orden público. }\end{array}$ & .48 & .90 & \\
\hline $\begin{array}{l}\text { 13. La protesta pública es uno de los mejores medios que } \\
\text { tiene la población para hacer valer sus legítimos derechos. } \\
\text { (Inverso) }\end{array}$ & .38 & .90 & \\
\hline $\begin{array}{l}\text { 14. Todo aquel que haya participado en una protesta } \\
\text { pública debería ser investigado por los servicios de } \\
\text { inteligencia del Estado. }\end{array}$ & .62 & .90 & \\
\hline
\end{tabular}


Ítems

\begin{tabular}{ccc} 
Correlación & Alfa de & Alfa de \\
ítem-test & Cronbach & Cronbach \\
corregida & $\begin{array}{c}\text { se elimina } \\
\text { el ítem }\end{array}$ & $\begin{array}{c}\text { de la escala } \\
\text { completa }\end{array}$ \\
\hline
\end{tabular}

15. Los medios de comunicación que apoyen protestas públicas deberían ser cerrados temporalmente.

16. La protesta pública es una de las mejores muestras de que existe legítima libertad de expresión en un país. (Inverso)

17. Se debe limitar drásticamente el accionar de aquellas ONG's (Organizaciones no gubernamentales) que apoyen protestas públicas.

18. No hay nada peor para una sociedad que la existencia de muchas protestas. Estas situaciones deberían eliminarse o reprimirse duramente.

19. Protestar públicamente es una de las maneras más legítimas para cambiar aquellas cosas con las que uno no está de acuerdo. (Inverso)

20. Cualquier persona que proteste públicamente debería ser considerado un delincuente.

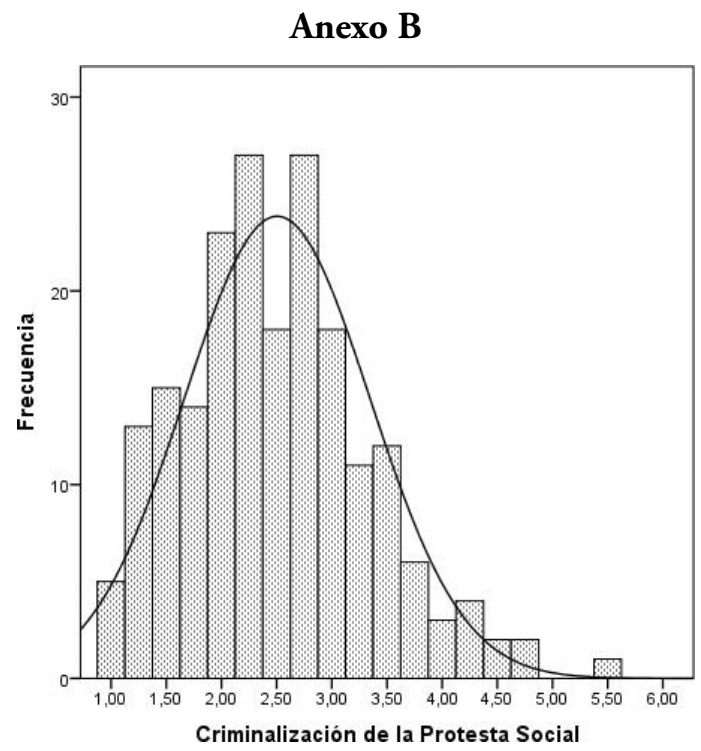

Gráfico 1. Histograma con curva normal de la variable "Criminalización de la protesta pública" 